\title{
Review Article \\ Ultrafast Four-Wave Optical Parametric Amplification in Transparent Condensed Bulk Media
}

\author{
Audrius Dubietis, Gintaras Tamošauskas, Gintaras Valiulis, and Algis Piskarskas \\ Department of Quantum Electronics, Vilnius University, Sauletekio Avenue 9, Building 3, 10222 Vilnius, Lithuania \\ Correspondence should be addressed to Audrius Dubietis, audrius.dubietis@ff.vu.lt
}

Received 28 March 2008; Accepted 15 May 2008

Recommended by Saulius Juodkazis

\begin{abstract}
We present a short overview of recent advances in ultrashort pulse four-wave optical parametric amplification in transparent condensed bulk media with Kerr nonlinearity. Highly efficient (10\% to 15\% pump-to-signal energy conversion) four-wave optical parametric amplification in water and fused silica is experimentally demonstrated. The amplification process highly benefits from 1-dimensional spatial soliton propagation regime, which sets in under proper combination of cylindrical beam focusing and noncollinear phase matching geometry with millijoule pumping. Under these operating conditions, strong four-wave coupling quenches catastrophic beam break-up and filamentation, and the setup operates reasonably below the damage threshold of the nonlinear medium. The proposed methodology offers a number of advantages as compared to guided-wave configurations in gaseous media.
\end{abstract}

Copyright (C) 2008 Audrius Dubietis et al. This is an open access article distributed under the Creative Commons Attribution License, which permits unrestricted use, distribution, and reproduction in any medium, provided the original work is properly cited.

\section{Introduction}

Four-wave mixing in transparent isotropic media with instantaneous nonlinear response (Kerr nonlinearity) is widely used in many fields of modern photonics: wide band telecommunications, imaging, metrology, and nonlinear optics [1]. In the ultrashort pulse regime, four-wave mixing manifests itself through generation of a broadband radiation, often termed white-light continuum, which can be produced in any media regardless of its physical state (e.g., solids, liquids, and gases) [2]. Generation of the whitelight continuum is associated with four-wave mixing, and, more precisely, with four-wave parametric amplification of the quantum noise, where multicolor radiation emerges at certain directions predefined by the phase-matching condition in bulk dispersive media. Far from frequency resonances, the nonlinear third-order susceptibility is rather low and therefore substantially high laser field intensity is required to induce an appreciable nonlinear response of the medium. On the other hand, high intensity gives rise to a manifold of competing nonlinear optical effects: stimulated Raman scattering, self- and cross-phase-modulation, and self-focusing, simultaneously occurring almost at the same intensity threshold [3]. In extended, wide-aperture bulk media, four-wave mixing is univocally linked to femtosecond filamentation phenomena, which represent an ultimate medium response to high optical intensity, and which recently experience a boost of interest associated with femtosecond pulse nonlinear optics $[4,5]$.

The discovery of the four-wave-mixing-driven optical parametric amplification dates back to the dawn of nonlinear optics [6]. However, real progress in the field by employing bulk transparent amplifying media has made a sensible breakthrough only recently. In 1997, Durfee et al. proposed a scheme for the four-wave parametric amplification in the ultrashort pulse regime, which exploits self-guided propagation of intense pump beam in noble gas-filled capillary waveguide [7]. The proposed technique successfully combined all the essential prerequisites for efficient energy exchange between the interacting waves through cubic response of the nonlinear medium: almost collinear phase-matching, high pump intensity, and long interaction length. Moreover, use of low dispersion gaseous media allowed to achieve broad amplification bandwidth $[8,9]$. More recently, four-wave optical parametric amplification 
was demonstrated in the filamentation regime, where the signal was amplified along the intense femtosecond filament in noble gas or air $[10,11]$ and has led to amplification of optical pulses with duration down to few optical-cycles [12].

In condensed bulk media, the conditions for efficient four-wave optical parametric amplification are much less favored [13]. Due to high material dispersion, nondegenerate four-wave parametric interactions could be phase matched only noncollinearly. This severely reduces useful beam overlap length in tight focusing geometry and implies using of short samples of the nonlinear media. On the other hand, applicable pump intensity is much limited by the occurrence of competing nonlinear effects: self-focusing, self- and cross-phase modulation, stimulated Raman scattering, and eventually, optical damage, which come into play almost at the same intensity threshold. So far, typical energy of the pulses amplified via parametric four-wave processes in bulk solid state media was in the order of $\sim 1 \mu \mathrm{J}$ in the visible spectral range [14] and few hundreds of $n J$ in the midinfrared spectral range [15]. A new route in practical implementation of efficient four-wave optical parametric amplification in water by means of cylindrical focusing and accessing 1-dimensional spatial soliton propagation regime, which benefits from extended beam overlap distance in the noncollinear phase-matching geometry, has been proposed [16].

In this paper, with an example of water as the nonlinear medium, we briefly overview the underlying principles that lead to efficient four-wave optical parametric amplification in bulk condensed media with Kerr nonlinearity. We demonstrate that the proposed methodology might be successfully applied to isotropic solid-state media (fused silica, in particular) to achieve the amplification of highenergy and broadband optical pulses. We also discuss further directions in development of the four-wave optical parametric amplifiers (FWOPAs) operating in the ultrashort pulse regime.

\section{Phase-Matching Geometry and Experimental Setup}

In isotropic medium, the cubic nonlinearity couples together four frequency components of the optical field through the induced nonlinear polarization. In the most general case, the nonlinear polarization might oscillate at positive 22 different frequencies and the observed process is termed four-wave mixing [17]. Confining ourselves to the particular case of interaction: four-wave parametric amplification with $\omega_{i}=$ $\omega_{p}+\omega_{p}-\omega_{s}$ (here subscripts $p, s, i$ stand for pump, signal, and idler waves), we will obtain a set of four equations describing the complex amplitude behavior versus propagation distance $z$ for the signal, idler, and two pump waves. For simplicity, if we assume that the two pump waves are identical, the interaction is fully described by the set of three coupled equations:

$$
\begin{aligned}
\frac{\partial A_{s}}{\partial z}+L_{s}\left(A_{s}\right)= & -i \sigma_{s} A_{p}^{2} A_{i}^{*} e^{-i(\Delta k z)}-i \sigma_{s}\left|A_{s}\right|^{2} A_{s} \\
& -i \sigma_{s}\left(2\left|A_{i}\right|^{2}+2\left|A_{p}\right|^{2}\right) A_{s}
\end{aligned}
$$

$$
\begin{aligned}
\frac{\partial A_{i}}{\partial z}+L_{i}\left(A_{i}\right)= & -i \sigma_{i} A_{p}^{2} A_{s}^{*} e^{-i(\Delta k z)}-i \sigma_{i}\left|A_{i}\right|^{2} A_{i} \\
& -i \sigma_{i}\left(2\left|A_{s}\right|^{2}+2\left|A_{p}\right|^{2}\right) A_{i}, \\
\frac{\partial A_{p}}{\partial z}+L_{p}\left(A_{p}\right)= & -i \sigma_{p} A_{s} A_{i} A_{p}^{*} e^{i(\Delta k z)}-i \sigma_{p}\left|A_{p}\right|^{2} A_{p} \\
& -i \sigma_{p}\left(2\left|A_{i}\right|^{2}+2\left|A_{s}\right|^{2}\right) A_{p},
\end{aligned}
$$

where $A_{j}$ is the complex amplitude with $j=p, s, i$ referring to pump, signal, and idler waves. $\sigma_{j}$ is the nonlinear coupling coefficient expressed through third-order nonlinear material susceptibility $\chi^{(3)}$ :

$$
\sigma_{j}=\frac{3}{8} \frac{\omega_{j}}{c n_{j}} \chi^{(3)}
$$

and $L_{j}$ is the linear propagation operator, which describes pulse propagation with the group velocity $u_{j}=\partial \omega_{j} / k_{j}$ and accounts for the group velocity dispersion, lateral walk-off, and diffraction:

$$
L_{j}=\frac{1}{u_{j}} \frac{\partial}{\partial t}-\frac{i}{2} g_{j} \frac{\partial^{2}}{\partial t^{2}}+\beta_{j} \frac{\partial}{\partial x}+\frac{i}{2 k_{j}} \nabla_{\perp},
$$

where $u_{j}$ is the pulse group velocity, $g_{j}$ is the group velocity dispersion coefficient, $\beta_{j}$ is the propagation angle with respect to the pump beam axis (in small-angle approximation it takes values of $\theta$ for the signal, $\phi$ for the idler, and 0 for the pump waves), $k_{j}=n_{j} \omega_{j} / c$ is the wave number, and $\nabla_{\perp}$ is the Laplace operator. The first term on the right-hand side of (1) describes the four-wave parametric amplification, while the second and third terms describe the effects of self-focusing and cross-phase modulation, respectively. For the pump beam, cross-phase modulation term is weak and can be neglected, whereas it plays a relevant role in spatial reshaping of the signal and idler waves. By contrast, selffocusing term is essential for the pump beam due to its high intensity, and may not be accounted in the equations describing propagation of the signal and idler waves. For what concerns the parametric amplification (first term on the right-hand side), the strength of induced nonlinear polarization, oscillating at frequency $\omega_{i}$, crucially depends on the phase mismatch of the interacting waves, which is expressed as $\Delta k=2 k_{p}-k_{s}-k_{i}$. Assuming normal group velocity dispersion, in collinear configuration $k_{p}+k_{p}$ is always smaller than $k_{s}+k_{i}$, therefore the condition of perfect phase matching $\Delta k=0$ is achievable in noncollinear geometry only. The wave-vector matching diagram for the four-wave parametric amplification is depicted in Figure 1. It considers an intense pump beam, represented by the sum of two collinear wave-vectors $\mathbf{k}_{p}$, signal $\left(\mathbf{k}_{s}\right)$, and idler $\left(\mathbf{k}_{i}\right)$ beams propagating at some angles $\theta$ and $\phi$ with respect to the pump beam propagation axis. The phase-matching condition is described by a vector equation: $2 \mathbf{k}_{p}=\mathbf{k}_{s}+\mathbf{k}_{i}$, which, projected in the transverse and longitudinal directions with respect to the pump beam axis, reads as

$$
\begin{gathered}
2 k_{p}=k_{s} \cos \theta+k_{i} \cos \phi, \\
k_{s} \sin \theta=k_{i} \sin \phi .
\end{gathered}
$$




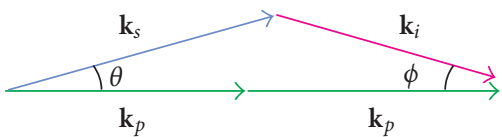

FIGURE 1: Wave-vector diagram describing the noncollinear fourwave parametric amplification. Color notation is for illustration of frequencies: $\omega_{s}>\omega_{p}>\omega_{i}$; note that the pump wave is composed of two photons of $\omega_{p}$.

After simple algebra, phase-matching angle $\theta$ between the pump and signal beams is expressed as

$$
\cos \theta=\frac{2 k_{p}^{2}+k_{s}^{2}-k_{i}^{2}}{4 k_{s} k_{p}},
$$

and which may be found with account for particular wavelengths and dispersion relation of the nonlinear medium used.

In the experiments, we used a commercial Nd:glass laser system (Twinkle, Light Conversion Ltd., Vilnius, Lithuania) which delivered 1-picosecond pulses at $1054 \mathrm{~nm}$ with energy up to $6 \mathrm{~mJ}$ at $10 \mathrm{~Hz}$ repetition rate. A fraction of either fundamental or second laser harmonics served as the pump beam for the FWOPA. The seed signal was delivered by a broadly (320-2600 nm) tunable, second-harmonic pumped optical parametric generator/amplifier system (Topas, Light Conversion Ltd.). Energy of the pump beam was adjusted by means of a half-wave plate and a thin-film polarizer, whereas energy of the seed signal was varied using a variable neutral density filter. Both pump and signal beams,parallel to each other, had linear polarization. The beam size of both pump and seed signal was aligned using suitable telescopes, and the temporal delay was matched using motorized mechanical delay lines. The beams were loosely focused onto the input face of a thick nonlinear medium by means of a cylindrical lens. The complete geometrical arrangement is illustrated in Figure 2, providing top and side views of the interaction. The focusing was performed in the vertical $(y)$ plane, while the beams were crossed in the horizontal $(x)$ plane, thus ensuring a good spatial overlap along entire sample length. Note that the input face of the nonlinear medium is placed exactly at the geometrical focus of the lens, and further 1-dimensional beam contraction along $y$-axis inside the medium is due to self-focusing effect. The beam crossing angle was calculated according to (5), with accounts for dispersive properties of the particular nonlinear medium and wavelengths used.

\section{Four-Wave Parametric Amplification in Water: Stable 1-Dimensional Spatial Solitons versus Multiple Filamentation}

The first set of experiments was performed using water as the nonlinear amplifying medium, which was contained within a variable-length cuvette with 1 -mm-thick fused silica windows. The construction of the cuvette allowed to vary its length and therefore to precisely monitor beam propagation dynamics inside the nonlinear medium. Second

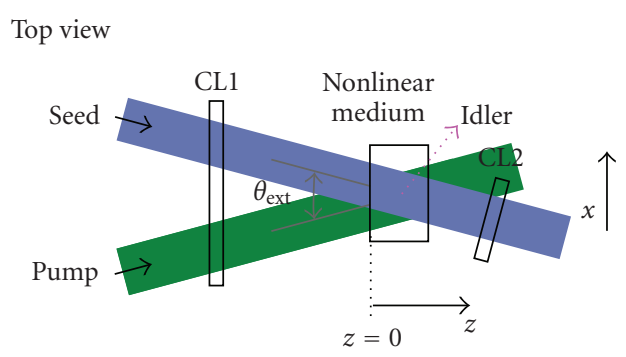

(a)

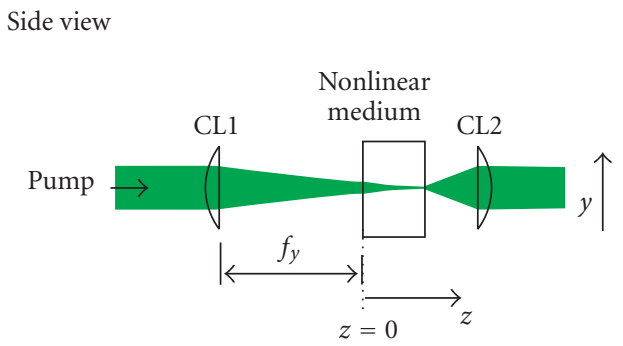

(b)

FIgURE 2: Geometrical beam arrangement in the FWOPA using cylindrical beam focusing. $\theta_{\text {ext }}$ is the external phase matching angle, $\theta_{\text {ext }} \approx n \theta$. CL1 and CL2 are cylindrical lenses for beam focusing and restoring, respectively.

harmonic of the laser served as the pump beam $\left(\lambda_{p}=\right.$ $527 \mathrm{~nm}$ ) and cylindrical focusing with $f_{y}=+500 \mathrm{~mm}, f_{x}=$ $\infty$ lens, yielded almost identical pump and seed signal beam dimensions of $1.2 \mathrm{~mm} \times 130 \mu \mathrm{m}$ at the input face of the water cuvette, as depicted in Figure 3(a). Energy of the pump beam was set to $E_{p}=75 \mu \mathrm{J}$, that is $\sim 65 P_{\text {cr }}$ in terms of critical power for self-focusing, where $P_{\mathrm{cr}}=3.77 \lambda_{p}^{2} /\left(8 \pi n_{0} n_{2}\right)=1.15 \mathrm{MW}$, with $n_{0}=1.33$ and $n_{2}=2.7 \times 10^{-16} \mathrm{~cm} / \mathrm{W}$ being linear and nonlinear refractive indexes of water, respectively. In the absence of the seed signal, highly elliptic pump beam undergoes gradual contraction in the $y$-plane, and after passing the nonlinear focus located at $z=25 \mathrm{~mm}$, it breaks up into an array of almost equally spaced multiple filaments, as shown in Figure 3(b). This break-up pattern is universal, and has been observed under different operating conditions and in a variety of nonlinear media [18-21]. Interestingly, further propagation of periodic multifilamentary structures in water gives rise to phase-matched stokes and antistokes emission at large angles mediated by the stimulated Raman scattering [22].

Injection of a weak signal beam $\left(\lambda_{s}=490 \mathrm{~nm}\right)$ at the phase-matching angle $\left(\theta_{\text {ext }}=1.0 \mathrm{deg}\right)$ immediately quenches the beam break-up and filament formation. In this case, pump beam still contracts along its short ( $y$-axis) direction and transforms into a uniform light stripe with $24 \mu \mathrm{m}$ FWHM diameter (Figure 3(c)). This structure propagates over a considerable distance without apparent diffraction (Figure 3(d)) inside the nonlinear medium, as measured by imaging the output face of the variable water cuvette onto a 10-bit dynamic range CCD camera (COHU-6612 linked to Spiricon LBA-400PC frame grabber). The diffraction-free 


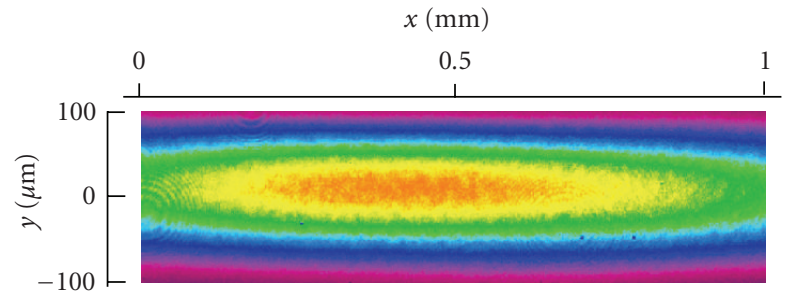

(a)

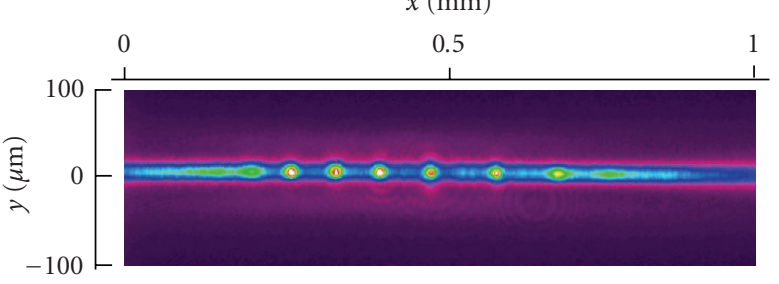

(b)

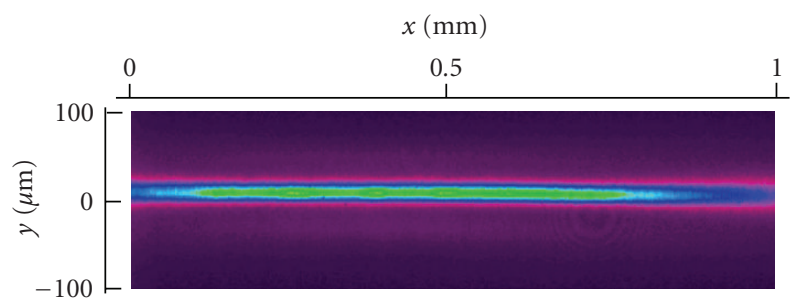

(c)

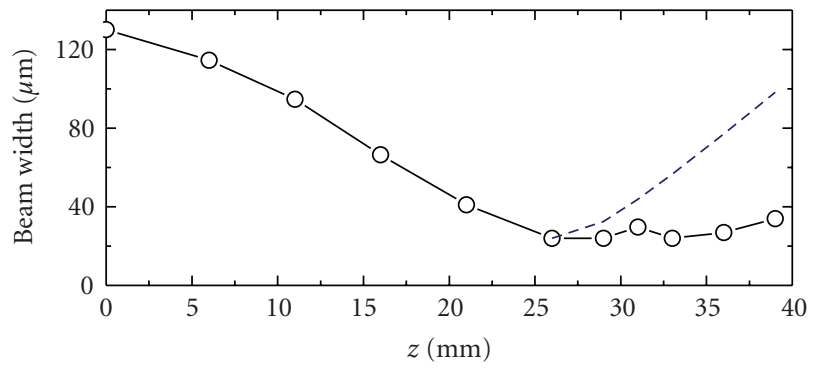

(d)

FIGURE 3: Near-field intensity profiles of the pump beam: (a) at the input and at the output of 40-mm-long water cell; (b) in the absence; (c) in the presence of the seed signal launched at the phase-matching angle; (d) measured FWHM diameter of the pump beam (circles and solid curve) versus propagation distance $z$ within the nonlinear medium and calculated diffraction spreading of the Gaussian beam of the same diameter (dashed curve).

propagation regime of the pump beam extends over a distance of $15 \mathrm{~mm}$, that is almost 4.5 times larger than the diffraction length for a Gaussian beam of the same diameter $\left(z_{R} \approx 3.3 \mathrm{~mm}\right)$. As a consequence, the pump beam preserves its high intensity and remains optimally overlapped with the noncollinear seed, thus providing an ideal condition for efficient four-wave parametric amplification to take place. The amplified signal $\left(\lambda_{s}=490 \mathrm{~nm}\right)$ and idler $\left(\lambda_{i}=\right.$ $570 \mathrm{~nm}$ ) beams exhibit high spatial quality and diffractionfree propagation as well, with their propagation being driven by spatial cross-phase modulation imposed by the intense pump beam. The overall observation suggests formation of stable 1-dimensional multicolor parametric solitons due to strong four-wave coupling. Here, off-axis propagating signal and idler waves act as a damping mechanism that efficiently suppresses any local spike that forms in the pump beam as a result of modulational instability. In our experiment, the soliton regime is sustained within a wide range of input parameters, with pump energy as high as $90 \mu \mathrm{J}$ and seed signal energy as low as $10 \mathrm{~nJ}$.

Figure 4 illustrates some of the autocorrelation measurements of the pump and signal pulses performed by a scanning autocorrelator, which also employs beam imaging. It is worth mentioning that in the absence of the seed signal, the pump breaks up not only in the spatial domain, but also in the temporal domain, resulting in pulse splitting within each individual filament (the result is presented elsewhere [23]). The split pulses further undergo complex propagation, similar to that observed in a single filament propagation dynamics [24]. However, the spatial as well as the temporal break-ups of the pump are quenched after injection of the seed pulse. The only observed slight deterioration of the pump pulse temporal profile (Figure 4(b)) occurs due to significant pump energy depletion. Under these settings, the amplified signal retains a clean Gaussian intensity profile as shown in Figure 4(d) and exhibits some slight pulsewidth narrowing from 0.7 picosecond to 0.5 picosecond as a result of parametric gain.

Summarizing the above observations, we conclude that noncollinear four-wave coupling is a highly efficient mechanism of the removal of space-time instabilities of 1dimensional spatial solitons in bulk nonlinear medium in the ultrashort pulse regime. It is worth mentioning that the stability problem of 1-dimensional spatial solitons in bulk media attracts a great attention from the fundamental and applied research (see [25] and references therein for an overview on the related topics) and stable 1-dimensional spatial solitons in the ultrashort pulse regime were never observed before.

\section{Energy Scaling}

Water FWOPA provides extremely high energy conversion efficiency from pump to parametric waves with a figure as high as $25 \%$ in the gain saturation regime with $E_{p}=$ $60 \mu \mathrm{J}$. The obtained result suggests a signal gain factor of $\sim 100$, with almost $15 \%$ pump-to-signal energy conversion. A distinct property of water FWOPA, operating at signal and idler wavelengths close to those of the pump, concerns a strong cascading effect, which manifests itself as an emergence of a series of frequency upshifted and downshifted components at the output, as shown in Figure 5.

The cascaded frequency components are generated through the multistep four-wave mixing processes $\mathbf{k}_{\mathbf{s} 1}=$ $2 \mathbf{k}_{\mathrm{s}}-\mathbf{k}_{\mathrm{p}}, \mathbf{k}_{\mathrm{s} 2}=2 \mathbf{k}_{\mathrm{s} 1}-\mathbf{k}_{\mathrm{s}}$, and so forth [14]. Here, genuine signal and idler waves and their cascaded counterparts, indicated by subscript numbering, serve as a new pump in the particular four-wave mixing process. The unequal number and uneven energy distribution between the generated frequency components can be explained by a particular 


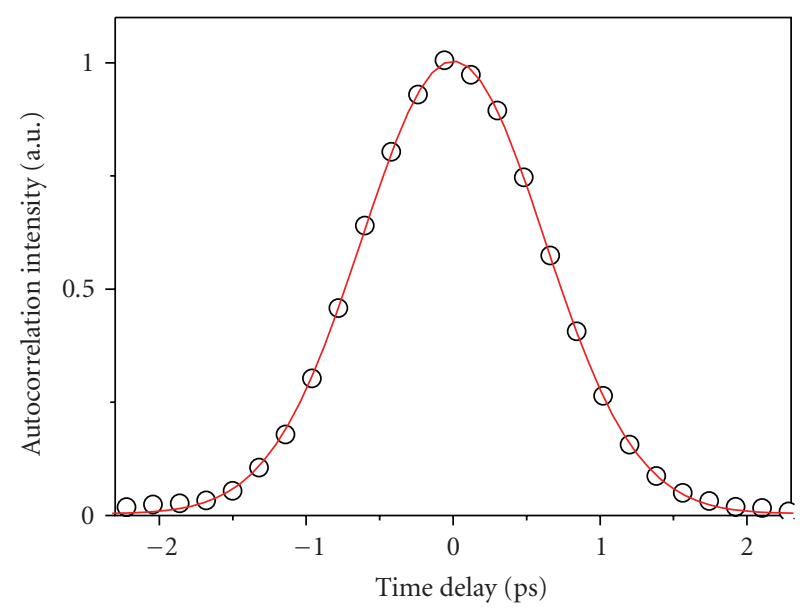

(a)

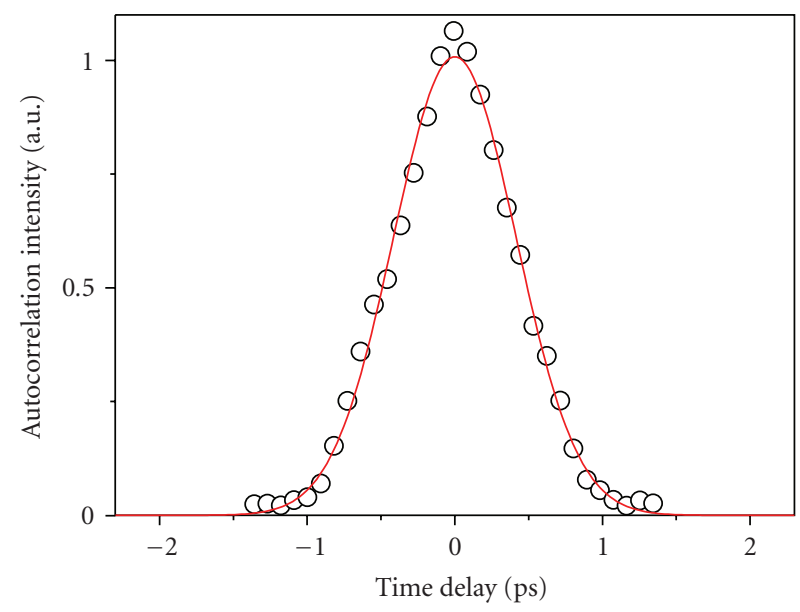

(c)

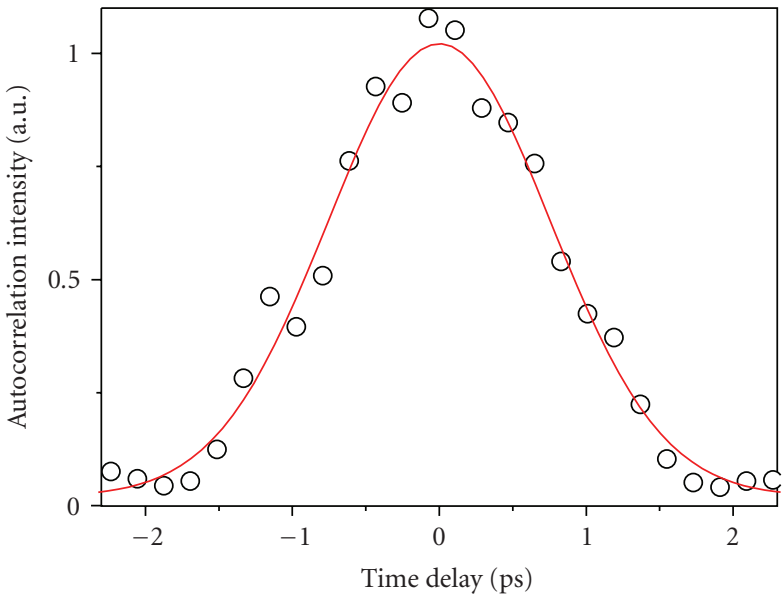

(b)

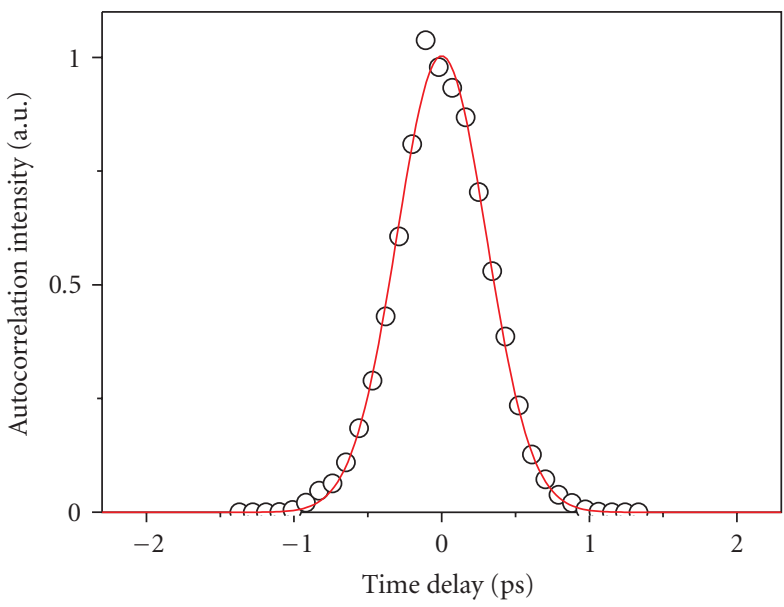

(d)

Figure 4: Normalized autocorrelation functions of (a) input pump pulse, (b) depleted pump pulse at the FWOPA output, (c) input seed signal, and (d) output amplified signal. Red curves represent a corresponding Gaussian fit. Output pulses were measured after propagation in 40-mm-long water cuvette, with $E_{p}=60 \mu \mathrm{J}$ and pump-to-signal conversion of $15 \%$.

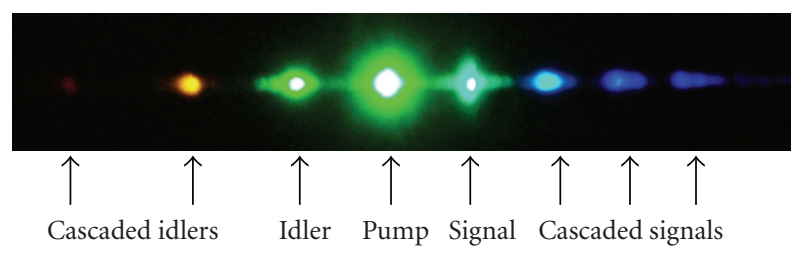

FigURE 5: FWOPA output imaged on the screen after the wavefront restoring cylindrical lens. The intense green spot in the center is the pump, the closest cyan and green spots are the signal and idler waves, respectively, while the remaining colorful spots are additional frequency components generated by the cascading.

phase-mismatch, which is established under predefined propagation angles and nontrivial impact of increasing linear absorption of water towards lower frequencies. The impact of these processes is investigated in more detail in $[16,23]$. Here we just mention that under present operating conditions, the cascaded components comprise only up to $20 \%$ of the total output energy and contribute with less than 5\% of the overall energy conversion. The number as well as energy of the cascaded components can be suppressed by tuning wavelength of the seed signal farther from that of the pump. The amplified signal energy is $10 \mu \mathrm{J}$, that compares to the signal energy obtained via four-wave mixing in noble gases and air under millijoule-pulse pumping [9-11]. Being different from guided-wave schemes, the pump beam size in 1-dimensional spatial soliton regime is not limited along one of the transverse dimensions (along $x$-axis), so present setup offers an excellent possibility of easy beam-size and energy scaling.

In order to demonstrate energy scaling capabilities of the FWOPA, we performed a second experiment on the four-wave optical parametric amplification in 12-mm-long fused silica (UV-grade, type KU-1) sample with pumping at fundamental laser frequency $\lambda_{p}=1054 \mathrm{~nm}$ and seeding with $\lambda_{p}=730 \mathrm{~nm}$. General settings in the interaction scheme are very similar as those depicted in Figure 2. The initial beam sizes of the pump and seed signal were expanded 


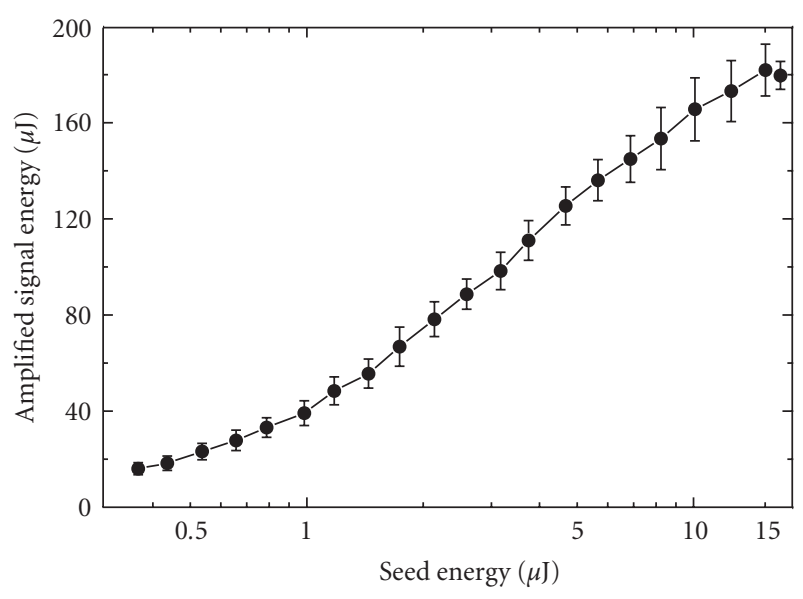

Figure 6: Amplified signal energy versus seed pulse energy measured at $E_{p}=1.8 \mathrm{~mJ}$ in 12 -mm-long fused silica sample.

by suitable telescopes, focused with $f_{y}=+750 \mathrm{~mm}, f_{x}=$ $\infty$ lens, yielding beam FWHM dimensions at the input face of the fused silica sample of $3.7 \mathrm{~mm} \times 195 \mu \mathrm{m}$ and $2.8 \mathrm{~mm}$ $\times 135 \mu \mathrm{m}$, respectively, and crossed at $\theta_{\mathrm{ext}}=1.59^{\circ}$ so as to ensure perfect phase matching.

The operational characteristics of the fused silica FWOPA at high energy pumping $\left(E_{p}=1.8 \mathrm{~mJ}\right)$ are depicted in Figure 6. By varying energy of the seed pulse from $0.3 \mu \mathrm{J}$ to $16 \mu \mathrm{J}$, we access the complete coverage of the amplification regimes: from the small signal gain up to the gain saturation. The highest small signal gain of $\sim 80$ is measured within seed energy interval of $0.3-1.5 \mu$ J. The gain factor gradually drops with increasing seed signal energy and saturates at $\sim 10$ with $15 \mu \mathrm{J}$ seed. In this condition, the largest amplified signal energy of $180 \mu \mathrm{J}$ is achieved, pointing to an appreciable pump-to-signal energy conversion efficiency of $9.2 \%$. As compared to previous results of water-based FWOPA, the present configuration is able to support high-energy amplification regime in condensed medium, and what is to the best of our knowledge the first demonstration of fourwave parametric amplification in transparent bulk medium with millijoule pumping. We also underline that such an impressive amplified signal energy extracted from the present scheme outreaches those produced so far in gaseous media by almost 20 times [7-12].

Figure 7 illustrates the central portion of the intensity profiles of input (seed) and output (amplified) signals, demonstrating how it reshapes into a smooth light stripe having $\sim 17 \mu \mathrm{m}$ FWHM diameter imposed by the propagation dynamics of the intense pump beam, which experiences 1-dimensional self-focusing. The pump beam, as measured at the output face of the fused silica sample, contracts along the $y$-axis to a dimension of $\sim 25 \mu \mathrm{m}$, whereas its wider $(x$ axis) dimension remains almost the same. The details of its spatial dynamics inside the sample were not captured due to fixed sample length, however, the observation of the spatial profile at the sample output suggests that no break-up into multiple filaments and no optical damage neither inside the sample nor on its output face there occurs at energies as

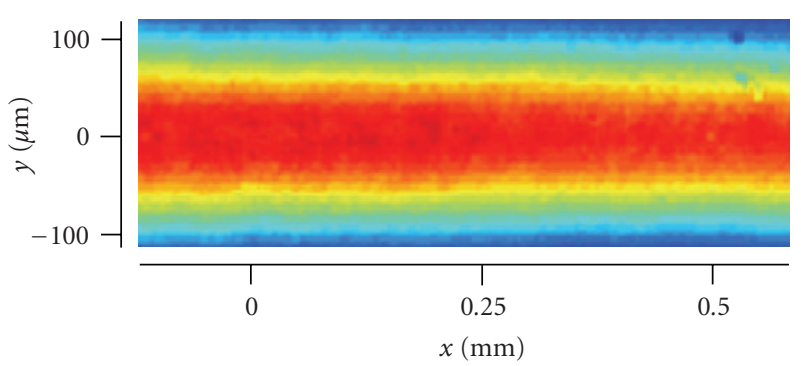

(a)

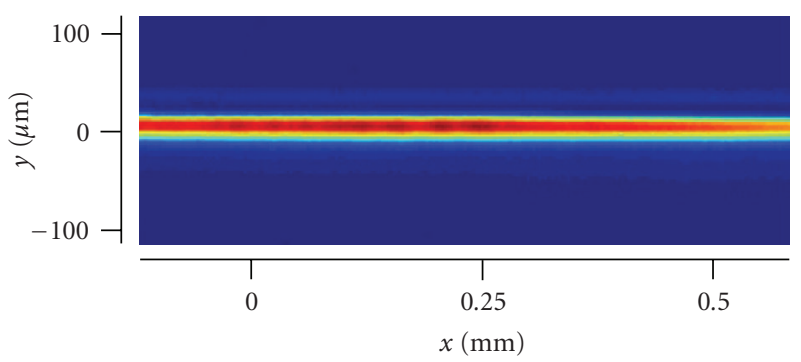

(b)

FIGURE 7: Near-field intensity profiles of (a) seed signal and (b) amplified signal at the fused silica FWOPA input and output, respectively.

high as $1.9 \mathrm{~mJ}$. We have evaluated the pump beam intensity of $210 \mathrm{GW} / \mathrm{cm}^{2}$ and $1.7 \mathrm{TW} / \mathrm{cm}^{2}$ assuming Gaussian spatial and temporal profiles at the input and output faces of the fused silica sample, respectively. The latter converts to a maximum fluence of $1.8 \mathrm{~J} / \mathrm{cm}^{2}$ without an account of possible multiphoton absorption and energy conversion into the parametric waves. We note that the damage fluence for fused silica is $2.5 \mathrm{~J} / \mathrm{cm}^{2}$ for 1-picosecond pulses [26], so our operating conditions are safely below the optical damage threshold.

\section{Broadband Four-Wave Optical Parametric Amplification}

One of the most important characteristics of any optical parametric amplifier is the gain bandwidth, which essentially determines its ability to amplify ultrashort light pulses. In the framework of the ultrashort pulse propagation, the amplification bandwidth is defined by the group-velocity $\left(v_{g}=\partial \omega / \partial k\right)$ differences between the signal and idler waves that convert to a wave-vector mismatch in the first-order approximation [27]:

$$
\Delta k=\left(\frac{1}{v_{g i}}-\frac{1}{v_{g s}}\right) \Delta \omega .
$$

In conventional three-wave optical parametric amplifiers, the broadband phase-matching is achieved by setting an appropriate angle between the pump and signal beams so as to match their wave-vector projections along the signal propagation direction: $v_{g s}=v_{g i} \cos \Theta[28]$, assuming that $v_{g s}<v_{g i}$ (that is the case of normal group-velocity dispersion) and where $\Theta$ denotes the angle between the 


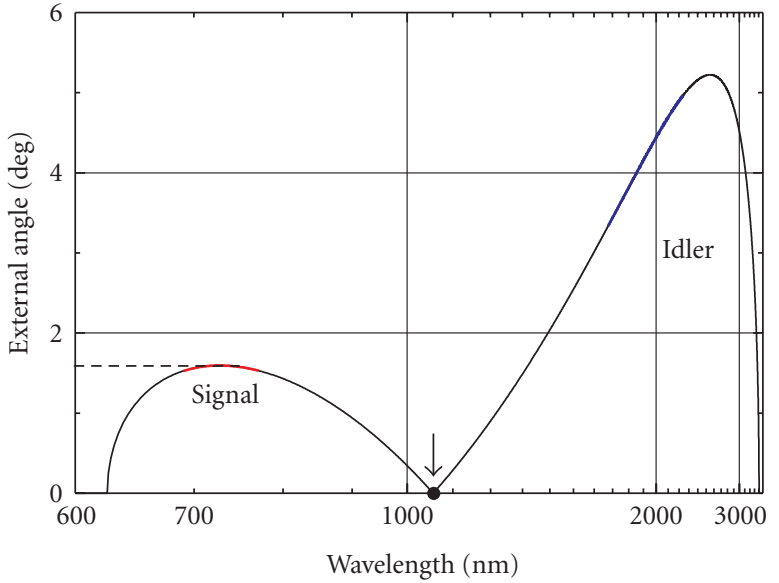

FIGURE 8: Phase-matching curve for the four-wave optical parametric amplification in fused silica. Wave-vectors of the pump with $\lambda_{p}=1054 \mathrm{~nm}$ lie on the propagation axis and are indicated by an arrow. Expected broadband amplification range for the signal is highlighted by the red curve, whereas the blue curve marks the corresponding idler wavelength range.

signal and corresponding idler frequency components. If the propagation direction of the signal is fixed, the angle $\Theta$ depends solely on its wavelength. The same considerations hold also for the four-wave optical parametric amplification. Figure 8 shows the phase matching curve in fused silica pumped by the fundamental harmonic of the Nd:glass laser $\left(\lambda_{p}=1054 \mathrm{~nm}\right)$. The character of the curve prompts that for signal wavelengths around $730 \mathrm{~nm}$, there exists an optimum beam crossing angle of $\theta_{\text {ext }}=1.59^{\circ}$, which keeps nearly constant over considerable signal wavelength range (indicated by a bold red curve). The corresponding idler waves (with wavelength from $1675 \mathrm{~nm}$ to $2240 \mathrm{~nm}$ ) fan out within a wide range of angles $\left(3.5^{\circ}-5^{\circ}\right.$, bold blue curve) when tuning the signal from $770 \mathrm{~nm}$ to $690 \mathrm{~nm}$.

Experimentally, in the first approach, we have scanned the amplification bandwidth of the FWOPA by launching a tunable narrow-band ( $3 \mathrm{~nm}$ FWHM) seed signal from the parametric generator/amplifier system Topas, maintaining a constant delay between the pump and seed signal pulses and fixed crossing angle. Wavelength of the seed signal was varied in $5 \mathrm{~nm}$ step from $680 \mathrm{~nm}$ to $820 \mathrm{~nm}$. The result is plotted in Figure 9, where the amplification bandwidth was measured in the small-signal $\left(E_{s}=1 \mu \mathrm{J}\right)$ amplification regime and in the gain saturation regime with $E_{s}=8 \mu \mathrm{J}$. Both scanned spectral gain profiles are quite similar, indicating FWHM amplification bandwidth of $\sim 75 \mathrm{~nm}$.

And finally, we have seeded the 12-mm-long fused silica FWOPA with a broadband white-light continuum signal with central wavelength at $740 \mathrm{~nm}$, which was generated by self-focusing of a pulsed Bessel beam. The broadband seed signal was produced as follows. The Gaussian output pulse of 1-picosecond duration at $\lambda=740 \mathrm{~nm}$ from the commercial optical parametric generator/amplifier system was directed to a BK7-glass axicon with apex angle of $175.4^{\circ}$, which produced a Bessel beam of the same pulsewidth, with

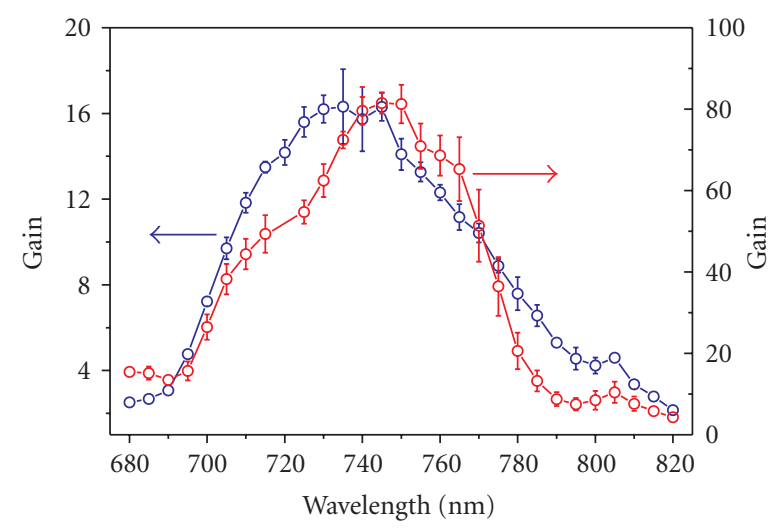

FIGURE 9: Scanning of the FWOPA amplification bandwidth with a narrow-band seed signal in the small signal amplification regime (red curve) and in the gain saturation regime (blue curve).

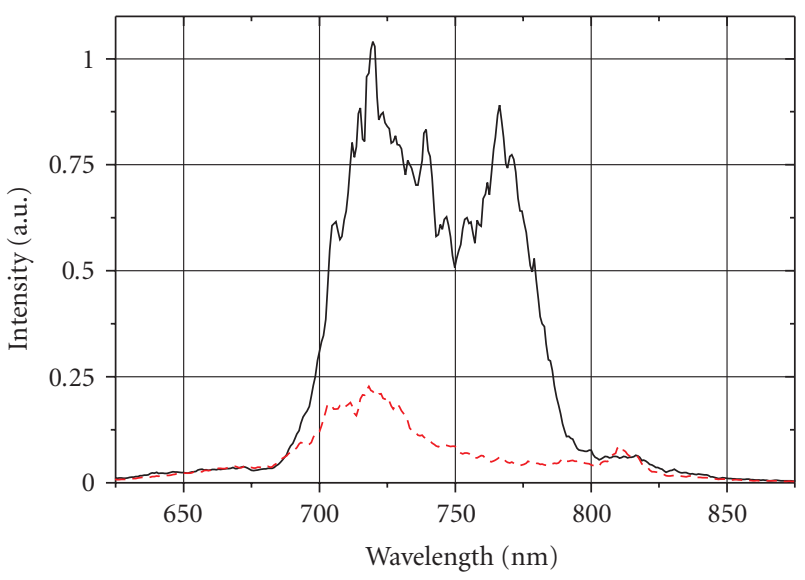

Figure 10: Amplified spectrum of the white-light continuum signal. Dashed curve indicates the input spectrum of broadband radiation.

diameter of the central core of $\sim 10 \mu \mathrm{m}$. The ultrashortpulsed Bessel with energy of $\sim 50 \mu \mathrm{J}$ was made to propagate in 20-mm-long fused silica sample, where it generated a narrow axial beam of high spatial quality with white-light spectral extent [29]. Here we note that the broadband axial radiation is easily separable from the intense spectral component of the pump Bessel beam by placing an iris aperture outside the Bessel zone. The spectral envelope of the white-light continuum radiation averaged over 100 laser shots is depicted by a dashed curve in Figure 10, and has a width of $170 \mathrm{~nm}$ estimated at $1 / e^{2}$ level. Spatial dimensions of the white-light continuum beam were matched to those of the pump in a similar manner as described in the previous experiments. The energy of the broadband seed signal at the FWOPA input was $0.75 \mu \mathrm{J}$.

The experimental conditions (focusing geometry and the input beam sizes) were identical to the previous experiment, and the beam crossing angle was kept at $\theta_{\text {ext }}=1.59^{\circ}$ so as to ensure broadband phase matching. Under these settings, the seed signal was amplified up to $11.5 \mu$ J energy with $1.8 \mathrm{~mJ}$ pump. We note here that the amplified signal energy could 
be considerably increased optimizing the seed pulse energy as demonstrated with a narrow-band seed experiment; see the results depicted in Figure 6. The spectrum of the amplified signal is shown in Figure 10. Its estimated FWHM bandwidth is $73 \mathrm{~nm}$, that is almost identical to that obtained with narrow-band signal scanning.

The pulse compression experiments with precise measurements of the pulse duration are in progress; we note, however, that the amplified spectrum width corresponds to a transform-limited pulse of $\sim 10$ fs duration.

\section{Conclusions and Outlook}

To summarize the results presented above, we have demonstrated an operating condition and methodology for highly efficient four-wave optical parametric amplification in isotropic transparent condensed media with Kerr nonlinearity. Our approach is based on cylindrical focusing geometry and utilization of 1-dimensional spatial soliton propagation regime, which comprise a complex solution for the problems generally encountered in the four-wave optical parametric amplification in bulk media so far as follows.

(i) Cylindrical focusing enables exact fulfillment of the noncollinear phase matching condition without reduction of the interaction length and allows high conversion efficiency to be achieved.

(ii) Stable 1-dimensional spatial soliton propagation regime ensures high beam quality and suppresses self-action effects in the space-time domain that lead to beam break-up, filamentation, and pulse splitting. In what follows, the setup operates reasonably below the damage threshold.

(iii) Being different from guided-wave configurations (hollow fibers and light filaments), 1-dimensional spatial solitons are not limited in aperture and therefore can be made to carry a large amount of energy, which can be easily scaled by increasing one (wider) transverse dimension without altering the propagation dynamics.

(iv) There is a great flexibility in achieving broad gain bandwidth in the four-wave parametric amplification process. It can be set either by making use of natural flattening of the noncollinear phase-matching curve or suitable signal pulse-front tilting (so-called achromatic phase matching) or eventually combining both possibilities altogether.

(v) Methodology of the four-wave optical parametric amplification is highly flexible in terms of wavelength range and therefore laser sources are to be used, and can be easily adapted to broad wavelength range from the UV up to mid-IR.

(vi) Condensed isotropic media have several advantages over gaseous media concerning their smaller dimensions and higher third-order nonlinearity. This property is essential for designing compact and high power four-wave optical parametric amplifiers.
The proposed four-wave optical parametric amplification methodology could be adapted to virtually all transparent media: solids, liquids, and gases and throughout a variety of wavelengths, especially those where conventional phasematching techniques based on material birefringence are of limited use or not applicable at all. Using second-harmonic pump pulses from Nd:glass or Ti:sapphire laser systems, four-wave optical parametric amplification in transparent bulk isotropic media may be readily shifted into the UV spectral region, where conventional phase matching techniques based on material birefringence suffer from many serious constraints. Employing achromatic phase matching technique [30] (that is imposing angular dispersion on the seed signal such that individual spectral components that comprise the broadband pulse are made to propagate at different angles to fulfill the phase-matching condition), it is possible to further extend the gain bandwidth and/or achieve tunability of the amplified signal. Taking an advantage of energy scaling capabilities, the proposed methodology might foresee the amplification of few-optical cycle pulses to millijoule energy. And finally, our finding of quenching the spontaneous nature of beam break-up and filamentation by means of launching a weak seed signal opens a new route towards controllable propagation of intense laser beams.

\section{Acknowledgments}

Authors acknowledge the financial support from the Lithuanian State Science and Studies Foundation (Project FORTAS, no. B-29/2008).

\section{References}

[1] R. R. Alfano, "The ultimate white light," Scientific American, vol. 295, no. 6, pp. 86-93, 2006.

[2] R. R. Alfano, The Supercontinuum Laser Source, Springer, New York, NY, USA, 2006.

[3] A. L. Gaeta, “Collapsing light really shines," Science, vol. 301, no. 5629, pp. 54-55, 2003.

[4] A. Couairon and A. Mysyrowicz, "Femtosecond filamentation in transparent media," Physics Reports, vol. 441, no. 2-4, pp. 47-189, 2007.

[5] L. Bergé, S. Skupin, R. Nuter, J. Kasparian, and J.-P. Wolf, "Ultrashort filaments of light in weakly ionized, optically transparent media," Reports on Progress in Physics, vol. 70, no. 10, pp. 1633-1713, 2007.

[6] R. L. Carman, R. Y. Chiao, and P. L. Kelley, "Observation of degenerate stimulated four-photon interaction and four-wave parametric amplification," Physical Review Letters, vol. 17, no. 26, pp. 1281-1283, 1966.

[7] C. G. Durfee III, S. Backus, M. M. Murnane, and H. C. Kapteyn, "Ultrabroadband phase-matched optical parametric generation in the ultraviolet by use of guided waves," Optics Letters, vol. 22, no. 20, pp. 1565-1567, 1997.

[8] C. G. Durfee III, S. Backus, H. C. Kapteyn, and M. M. Murnane, "Intense 8 -fs pulse generation in the deep ultraviolet," Optics Letters, vol. 24, no. 10, pp. 697-699, 1999.

[9] A. E. Jailaubekov and S. E. Bradforth, "Tunable 30femtosecond pulses across the deep ultraviolet," Applied Physics Letters, vol. 87, no. 2, Article ID 021107, 3 pages, 2005. 
[10] F. Théberge, N. Aközbek, W. Liu, A. Becker, and S. L. Chin, "Tunable ultrashort laser pulses generated through filamentation in gases," Physical Review Letters, vol. 97, no. 2, Article ID 023904, 4 pages, 2006.

[11] T. Fuji, T. Horio, and T. Suzuki, "Generation of 12 fs deepultraviolet pulses by four-wave mixing through filamentation in neon gas," Optics Letters, vol. 32, no. 17, pp. 2481-2483, 2007.

[12] T. Fuji and T. Suzuki, "Generation of sub-two-cycle midinfrared pulses by four-wave mixing through filamentation in air," Optics Letters, vol. 32, no. 22, pp. 3330-3332, 2007.

[13] A. Penzkofer and H. J. Lehmeier, "Theoretical investigation of noncollinear phase-matched parametric four-photon amplification of ultrashort light pulses in isotropic media," Optical and Quantum Electronics, vol. 25, no. 11, pp. 815-844, 1993.

[14] H. Crespo, J. T. Mendonça, and A. Dos Santos, "Cascaded highly nondegenerate four-wave-mixing phenomenon in transparent isotropic condensed media," Optics Letters, vol. 25, no. 11, pp. 829-831, 2000.

[15] H.-K. Nienhuys, P. C. M. Planken, R. A. van Santen, and H. J. Bakker, "Generation of mid-infrared pulses by $X^{(3)}$ difference frequency generation in $\mathrm{CaF}_{2}$ and $\mathrm{BaF}_{2}$," Optics Letters, vol. 26, no. 17 , pp. 1350-1352, 2001.

[16] A. Dubietis, G. Tamošauskas, P. Polesana, et al., "Highly efficient four-wave parametric amplification in transparent bulk Kerr medium," Optics Express, vol. 15, no. 18, pp. 11126 11132, 2007.

[17] R. W. Boyd, Nonlinear Optics, Academic Press, San Diego, Calif, USA, 2003.

[18] H. Schroeder and S. L. Chin, "Visualization of the evolution of multiple filaments in methanol," Optics Communications, vol. 234, no. 1-6, pp. 399-406, 2004.

[19] T. D. Grow and A. L. Gaeta, "Dependence of multiple filamentation on beam ellipticity," Optics Express, vol. 13, no. 12, pp. 4594-4599, 2005.

[20] M. Centurion, Y. Pu, and D. Psaltis, "Self-organization of spatial solitons," Optics Express, vol. 13, no. 16, pp. 6202-6211, 2005.

[21] A. Dubietis, E. Kučinskas, and G. Tamošauskas, "Formation of periodic multifilamentary structures by use of highly elliptical light beams," Lithuanian Journal of Physics, vol. 47, pp. 27-30, 2007.

[22] D. Faccio, A. Dubietis, G. Tamošauskas, et al., "Phase- and group-matched nonlinear interactions mediated by multiple filamentation in Kerr media," Physical Review A, vol. 76, no. 5, Article ID 055802, 4 pages, 2007.

[23] H. Valtna, A. Dubietis, G. Tamošauskas, et al., "Efficient fourwave parametric amplification and spatial soliton generation in transparent isotropic medium with Kerr nonlinearity," Lithuanian Journal of Physics, vol. 47, pp. 403-410, 2007.

[24] A. Matijosius, J. Trull, P. Di Trapani, et al., "Nonlinear spacetime dynamics of ultrashort wave packets in water," Optics Letters, vol. 29, no. 10, pp. 1123-1125, 2004.

[25] C. Anastassiou, M. Soljačić, M. Segev, et al., "Eliminating the transverse instabilities of Kerr solitons," Physical Review Letters, vol. 85, no. 23, pp. 4888-4891, 2000.

[26] B. C. Stuart, M. D. Feit, A. M. Rubenchik, B. W. Shore, and M. D. Perry, "Laser-induced damage in dielectrics with nanosecond to subpicosecond pulses," Physical Review Letters, vol. 74, no. 12, pp. 2248-2251, 1995.

[27] G. Cerullo and S. De Silvestri, "Ultrafast optical parametric amplifiers," Review of Scientific Instruments, vol. 74, no. 1, pp. $1-18,2003$.
[28] T. Wilhelm, J. Piel, and E. Riedle, "Sub-20-fs pulses tunable across the visible from a blue-pumped single-pass noncollinear parametric converter," Optics Letters, vol. 22, no. 19, pp. 1494-1496, 1997.

[29] A. Dubietis, P. Polesana, G. Valiulis, A. Stabinis, P. Di Trapani, and A. Piskarskas, "Axial emission and spectral broadening in self-focusing of femtosecond Bessel beams," Optics Express, vol. 15, no. 7, pp. 4168-4175, 2007.

[30] O. E. Martinez, "Achromatic phase matching for second harmonic generation of femtosecond pulses," IEEE Journal of Quantum Electronics, vol. 25, no. 12, pp. 2464-2468, 1989. 